\title{
17Beta-estradiol Stimulates Glucose Uptake Through Estrogen Receptor and AMP-activated Protein Kinase Activation in C2C12 Myotubes (Korean J Obes 2016;25:190-6)
}

\author{
Hyo-Bum Kwak* \\ Department of Kinesiology, Inha University, Incheon, Korea
}

Obesity induced by high calorie/fat diet and physical inactivity can result in serious health problems including chronic diseases such as cardiovascular disease and type 2 diabetes, which are associated with insulin resistance. ${ }^{1}$ Insulin resistance can affect skeletal muscle, liver, $\beta$-cells, fat cells, the gastrointestinal track, kidneys, and brain. ${ }^{2}$ Among these, skeletal muscle insulin resistance caused by obesity or type 2 diabetes is manifested as decreased glucose uptake because skeletal muscle comprises $40-50 \%$ of the total human body mass. It has been demonstrated by numerous studies that skeletal muscle insulin resistance is associated with mitochondrial dysfunction including 1) reduced fatty acid oxidation and increased accumulation of lipid intermediates (e.g., FA-CoA, DAG, ceramide $)^{3,4}, 2$ ) increased mitochondrial overload and incomplete fatty acid oxidation ${ }^{5}$, and 3) increased mitochondrial oxidative stress (e.g., $\mathrm{H}_{2} \mathrm{O}_{2}$ ) in skeletal muscle. ${ }^{6,7}$

Lee et al. ${ }^{8}$ investigated 17 beta-estradiol-stimulated glucose uptake through estrogen receptor and AMP-activated protein kinase (AMPK) activation in C2C12 myotubes. They showed that 17beta-estradiol treatment increased the protein levels of pAMPK and pACC (acetyl-CoA carboxylase) in C2C12 myotubes. Also, they reported that 17beta-estradiol treatment upregulated the mRNA expression of peroxisome proliferator-activated receptor $\gamma$ coactivator $1 \alpha($ PGC-1 1$)$, carnitine palmitoyltransferase 1 (CPT1), uncoupling protein 2 (UCP2), and glucose transporter 4 (GLUT4) in $\mathrm{C} 2 \mathrm{C} 12$ myotubes. Furthermore, they demonstrated that 17beta-estradiol treatment stimulated glucose uptake in $\mathrm{C} 2 \mathrm{C} 12$ myotubes by activating estrogen receptor (ER) and AMPK pathways, suggesting that estrogen treatment might protect against the risk of type 2 diabetes and metabolic syndrome in postmenopausal women with estrogen deficiency. The findings are very interesting and therapeutically significant in obesity, type 2 diabetes, and metabolic syndrome. This study suggests AMPK as a highly attractive target for the development of strategies to curb the prevalence and costs of obesity and type 2 diabetes. However, they could not determine the potential mechanism(s) through which 17beta-estradiol stimulated the expression of genes associated with glucose uptake in $\mathrm{C} 2 \mathrm{C} 12$ myotubes.

Mitochondria may be associated with a potential mechanism by which 17beta-estradiol treatment stimulates glucose uptake in $\mathrm{C} 2 \mathrm{C} 12$ myotubes because this study reported that PAMPK and PGC-1 1 were increased by 17 beta-estradiol treatment in $\mathrm{C} 2 \mathrm{C} 12$ myotubes. It is known that AMPK stimulates mitochondrial bio- 
genesis by regulating PGC-1 $\alpha$, which in turn promotes gene transcription in mitochondria and the anti-oxidant defense system. ${ }^{9}$ Recent evidence suggests that the accumulation of fatty acid metabolites associated with mitochondrial dysfunction including fatty acid-CoA (FA-CoA), diacylglycerol (DAG), and ceramide directly or indirectly alter the insulin signaling pathway. ${ }^{3}$ In normal mitochondria, free fatty acids produce acetyl-CoA during $\beta$-oxidation, which undergoes additional processes including the Krebs cycle and electron transport chain (ETC) in the mitochondrial matrix, leading to $\mathrm{CO}_{2}$ production in the Krebs cycle and ATP generation in the ETC. However, excess free fatty acid intake in obesity (i.e., excessive $\beta$-oxidation) induces a lipid burden on mitochondria (called 'mitochondrial overload'), and the mitochondria produce partially oxidized fatty acids such as acylcarnitine (called incomplete fatty acid oxidation). This incomplete fatty acid oxidation may contribute to lipid-induced impairments in insulin action. ${ }^{5}$ Furthermore, emerging evidence indicates that overnutrition results in elevated mitochondrial oxidative stress (i.e., reactive oxygen species; ROS), which is the primary factor in the development of insulin resistance in skeletal muscle. ${ }^{6,7}$ Therefore, the authors note that they will investigate the role of estrogen and mitochondria, which is associated with glucose uptake in skeletal muscle. Moreover, the effects of estrogen on obesity-induced insulin resistance in skeletal muscle should be determined as a future direction.

One more intriguing part in this study is that 17 beta-estradiol treatment stimulates the upregulation of ACC, CPT1, UCP2 as well as AMPK in C2C12 myotubes, which are associated with fatty acid oxidation. It is well known that one of the important pathways in AMPK's regulation of fatty acid metabolism is the phosphorylation and inhibition of ACC. ACC converts acetyl-CoA to maloylCoA, an inhibitor of CPT- $1 .^{10}$ Therefore, we expect that 17 beta-estradiol treatment may phosphorylate both AMPK and ACC, and the inactivation of ACC may result in increased CPT-1, leading to improved fatty acid transport in mitochondria and subsequent fatty acid oxidation. Accordingly, the authors should measure the effects of estrogen on fatty acid oxidation in skeletal muscle to determine the role of estrogen in the fatty acid oxidation pathway.

\section{CONFLICTS OF INTEREST}

The author has no conflicts of interest to declare.

\section{REFERENCES}

1. Abdul-Ghani MA, DeFronzo RA. Pathogenesis of insulin resistance in skeletal muscle. J Biomed Biotechnol 2010;2010: 476279 .

2. DeFronzo RA. From the triumvirate to the ominous octet: a new paradigm for the treatment of type 2 diabetes mellitus. Diabetes 2009;58:773-95.

3. Lowell BB, Shulman GI. Mitochondrial dysfunction and type 2 diabetes. Science 2005;307:384-7.

4. Holland WL, Brozinick JT, Wang LP, Hawkins ED, Sargent KM, Liu Y, et al. Inhibition of ceramide synthesis ameliorates glucocorticoid-, saturated-fat-, and obesity-induced insulin resistance. Cell Metab 2007;5:167-79.

5. Koves TR, Ussher JR, Noland RC, Slentz D, Mosedale M, Ilkayeva $\mathrm{O}$, et al. Mitochondrial overload and incomplete fatty acid oxidation contribute to skeletal muscle insulin resistance. Cell Metab 2008;7:45-56.

6. Anderson EJ, Lustig ME, Boyle KE, Woodlief TL, Kane DA, Lin CT, et al. Mitochondrial H2O2 emission and cellular redox state link excess fat intake to insulin resistance in both rodents and humans. J Clin Invest 2009;119:573-81.

7. Houstis N, Rosen ED, Lander ES. Reactive oxygen species have a causal role in multiple forms of insulin resistance. $\mathrm{Na}$ ture 2006;440:944-8.

8. Lee KH, Jo KJ, Kim JY, Baik HW, Lee SK. 17Beta-estradiol stimulates glucose uptake through estrogen receptor and AMP-activated protein kinase activation in $\mathrm{C} 2 \mathrm{C} 12$ myotubes. Korean J Obes 2016;25:190-6.

9. Jäger S, Handschin C, St-Pierre J, Spiegelman BM. AMP-activated protein kinase (AMPK) action in skeletal muscle via direct phosphorylation of PGC-1alpha. Proc Natl Acad Sci U S A $2007 ; 104: 12017-22$.

10. Saha AK, Ruderman NB. Malonyl-CoA and AMP-activated protein kinase: an expanding partnership. Mol Cell Biochem 2003;253:65-70. 